\title{
Lipid Lowering Potentials and Phytochemical Properties of Colocasia esculenta Leaf Extract in Rats
}

\author{
Frank Uchenna Eneh*, Uchechukwu Chibuzo Ogbodo, Chibuike Johnson Ogbu
}

Department of Applied Biochemistry, Nnamdi Azikiwe University, Awka, Anambra State, Nigeria

\author{
Email address: \\ fu.eneh@unizik.edu.ng (F. U. Eneh) \\ ${ }^{*}$ Corresponding author
}

\section{To cite this article:}

Frank Uchenna Eneh, Uchechukwu Chibuzo Ogbodo, Chibuike Johnson Ogbu. Lipid Lowering Potentials and Phytochemical Properties of Colocasia esculenta Leaf Extract in Rats. Advances in Biochemistry. Vol. 8, No. 1, 2020, pp. 16-20. doi: 10.11648/j.ab.20200801.13

Received: February 9, 2020; Accepted: February 26, 2020; Published: March 10, 2020

\begin{abstract}
Hyperlipidemia has remained a major risk factor in the pathophysiology of atherosclerosis and other cardiovascular diseases and research efforts have been geared toward proffering remedies through diet and nutrition inherent in potent herbaceous plants. This study therefore investigated the effect of ethanol extract of the leaves of Colocasia esculenta on the serum lipid profile of male Albino rats as well as phytochemicals of ethnomedicinal importance. A total of 18 rats divided into three groups of 6 rats each were engaged in the investigation. The first group (baseline) was sacrificed after purchase; second group (control) was fed rat chow, and the third group (test) was fed a composite feed containing rat chow and 5\% extract of C. esculenta leaves. The lipid profile total cholesterol (TC), triacylglycerols (TAG), Low Density Lipoprotein (LDL), High Density Lipoprotein (HDL) and Very Low Density Lipoprotein (VLDL) of the rats were assayed after 21 days of feeding and the result showed a significant difference $(p<0.05)$ between the test and control groups for the lipids assayed. The extracts of $C$. esculenta reduced TC and LDL levels significantly $(p<0.05)$ from $4.01 \pm 2.28$ in control to $0.31 \pm 0.25 \mathrm{mmol} / \mathrm{L}$ in the test for TC and then from $1.53 \pm 1.19$ in the control to $0.07 \pm 0.04 \mathrm{mmol} / \mathrm{L}$ in the test for LDL. This indicates that the leaves of this plant have a very high tendency to be protective against cardiovascular diseases. However, there was a marked increase in the TAG level of the test group $(2.37 \pm 0.84 \mathrm{mmol} / \mathrm{L})$ compared to the control group $(0.22 \pm 0.18 \mathrm{mmol} / \mathrm{L})$. This was nevertheless found to be not significant $(p>0.05)$ but indicates the possibility for enhancement of TAG synthesis by the leaves of $C$. esculenta. Phytochemical screening showed a rich array of plant metabolites including flavonoids, saponins, tannins and alkaloids which may contribute to the hypolipidemic effects of ethanol extract of the plant. Further investigations may require identifying and characterizing the phytochemicals responsible for the overall role of the plant in folklore medicine.
\end{abstract}

Keywords: Colocasia esculenta, Leaves, Serum, Lipid Profile, Rats, Hyperlipidemia

\section{Introduction}

Hyperlipidemia is a major modifiable risk factor in the pathogenesis of most cardiovascular diseases (CVDs). CVDs account for approximately $71 \%$ mortality attributed to communicable diseases globally with an estimated 17.9 million persons representing $31 \%$ of all global deaths occurring only in 2016 [1]. Of these deaths, $85 \%$ were due to heart attack and stroke [1]. Hyperlipidemia refers to a heterogeneous group of disorders characterized by an excess of blood lipids such as cholesterol, phospholipids and triglycerides. These blood lipids, which are water insoluble organic compounds are essential for many normal physiological activities of living organisms as important components of cell membranes and energy stores. They also play significant roles as enzyme cofactors, hormones and intracellular messengers [2]. However, it has been evidenced by several research findings that dysfunctional lipid metabolism is linked to a number of diseases including type 2 diabetes mellitus, coronary artery disease, nonalcoholic fatty liver diseases and cancer [3, 4]. Consequently, supply of lipids for biological activity needs to be adequate and regulated, hence the need to monitor elevated or decreased levels of specific blood lipid types to serve as indicators in accessing lipid metabolic disorders [5]. Recently, increasing numbers of people in the developing countries, including 
Nigeria are becoming more vulnerable to the effects of hyperlipidemia due to poor lifestyle and dietary habits.

Current recommended treatment strategies are directed at improving lifestyle including reducing tobacco smoke intake, harmful use of alcohol and unhealthy diet with increasing physical activity. And upon the thrust of improving healthy diet hangs consumption of indigenous fruits and vegetables that may have lipid lowering effects on the cardiovascular system. In the light of this, attention has been drawn to our plant leaves which form part of our local diets to assess their efficacy in the treatment of cardiovascular diseases. One of these is Colocasia esculenta leaf.

C. esculenta also known as cocoyam, belongs to the family of Araceae. It is largely grown for its edible corms, cormels and leaves for use by subsistence farmers [6]. In the SouthEast region of Nigeria, the leaves of $C$. esculenta after drying are used for sauces and widely consumed by the people. It is called "Mpotoede" by the South easterners of Nigeria who widely consume it. The corms of $C$. esculenta have been reported to contain nutrients like carbohydrates, starch, protein, fiber, minerals and secondary metabolites as flavonoids, alkaloids, glycosides and tannins [7, 8,]. The leaves have also been studied to show hypoglycemic [9], anti-cancer [10], hypolipidemic [11, 12], anti-inflammatory [13], anti-anemic, [14], antioxidant [15], hepatoprotective [16] and neuro-pharmacological activities [17].

Regardless these varied reports, there appears to be dearth of information on the effects of diet supplementation with ethanol extract of the plant leaves grown on Nigerian soil on serum lipid profile of male albino rats. Hence, the study was designed to address this gap with a view to finding a therapeutic application of the leaves of $C$. esculenta in the management and treatment of cardiovascular diseases.

\section{Materials and Methods}

\subsection{Sample Collection and Preparation}

Fresh leaves of $C$. esculenta for the purpose of the study were purchased from a farm in Emene, Enugu State, Nigeria and authenticated by a taxonomist of the Department of Botany, Nnamdi Azikiwe University, Awka, Nigeria. The leaves were washed with distilled water and dried under room temperature for 2 weeks. The leaves were then ground into fine powder using an electric blender and $5 \mathrm{~g}$ of the ground leaves immersed in $70 \%$ ethanol for 24 hours. Thereafter, the mixture was filtered and ethanol evaporated using a Soxhlet apparatus. The extract obtained was used to supplement standard rat chows to serve as experimental feed.

\subsection{Experimental Feed Formulation}

The leaf extract of $C$. esculenta and standard rat chow were mixed in a ratio of 5:95 to obtain the experimental diet. The pelletized rat chow (Vital Grower's Feed) was composed of crude protein $(13 \%)$, fat $(8 \%)$, crude fiber $(15 \%), \mathrm{Ca}$ $(0.9 \%), \mathrm{P}(0.35 \%)$, energy $(2600 \mathrm{Kcal} / \mathrm{kg})$.

\subsection{Animal Handling and Grouping}

A total of 18 Wistar albino rats (8-13 weeks) purchased from a commercial Animal Farm situated at Mgbakwu, Awka North Local Government Area, Anambra State were used in the study. The animals were handled in accordance with National Institute of Health $(\mathrm{NIH})$ ethical guidelines for the Care and Use of Laboratory Animals. The rats were divided into 3 groups (Group 1-3) of 6 rats each (namely baseline, control and test groups). The first group (Group 1) served as baseline; they were sacrificed immediately after purchase and blood collected via cardiac puncture. The blood samples were centrifuged and the sera obtained were used for baseline lipid profile analyses. Group 2 was fed only the Vital Grower's mash for 21 days and served as the experimental control. The final group (test group) received the compounded diet of 5\% leaf extract of C. esculenta and 95\% standard rat chow for 21 days. All animals were given food and water ad libitum.

\subsection{Sample Collection for Biochemical Investigation}

After 21 days of feeding the animals in the test and control groups, they were fasted overnight for 12 hours, weighed and anaesthetized using chloroform-soaked cotton wool in a plastic container. Blood samples were collected from the rats via cardiac puncture and subsequently centrifuged at 1000 rpm for 30 minutes to obtain the sera which was further subjected to lipid profile analyses.

\subsection{Lipid Profile Investigations}

Serum Total cholesterol, Triacylglycerol (TAG), Low Density Lipoprotein (LDL), High Density Lipoprotein (HDL) and Very Low Density Lipoprotein (VLDL) were determined using standard commercial Autochemistry Analyzer (Mindray BA-88, China) according to the principle of methods described in a previous work published in 2012 [18].

\subsection{Qualitative Phytochemical Screening of C. esculenta}

The ethanol plant extract was screened for the presence of alkaloids, flavonoids, saponins, cardiac glycosides, tannins, sterols, reducing sugars and terpenoids according to methods of Harborne [19].

\subsection{Statistical Analysis}

All statistical analyses were performed using SPSS IBM version 23.0 (SPSS Inc., Illinois Chicago, USA). One-way Analysis of Variance (ANOVA) was used to check the relationship across the group means. Data were presented as mean + SD with $p<0.05$ at $95 \%$ confidence level considered significant.

\section{Results}

The effects of the ethanol extract of the leaves of $C$. esculenta on serum lipid profile of rats fed supplemented 
feed are shown in Table 1.

In the present study, from the results obtained a significant decrease $(p<0.05)$ in mean values of total cholesterol, HDLcholesterol and LDL-cholesterol of the test group was indicated when compared with control and baseline. The serum cholesterol mean determination for baseline, control and test groups was $3.399 \mathrm{mmol} / \mathrm{L}, 4.019 \mathrm{mmol} / \mathrm{L}$ and $0.317 \mathrm{mmol} / \mathrm{L}$ respectively. The cholesterol level was noted significantly decrease $(p<0.05)$ in the test group when compared with the control.

The results also showed that there was significant decrease $(p<0.05)$ in the concentration of serum HDL level of the test group $(0.223 \mathrm{mmol} / \mathrm{L})$ compared to the control $(3.001 \mathrm{mmol} / \mathrm{L})$ and baseline $(0.837 \mathrm{mmol} / \mathrm{L})$ groups. The results for triacylglycerols showed a significant increase $(p<0.05)$ in test groups with a mean value of $2.394 \mathrm{mmol} / \mathrm{L}$, $3.856 \mathrm{mmol} / \mathrm{L}$ and $0.221 \mathrm{mmol} / \mathrm{L}$ for the test, baseline and control groups respectively.
There was a significant decrease $(p<0.05)$ in the concentration of VLDL-cholesterol as seen in control $(0.044 \mathrm{mmol} / \mathrm{L})$ and test $(0.461 \mathrm{mmol} / \mathrm{L})$ groups when compared to the baseline group with a concentration of $0.077 \mathrm{mmol} / \mathrm{L}$

Also the serum TAG levels were found to be increased in the test animals against the control animals. These values, however, were not significantly different $(p>0.05)$.

Table 2 shows results of preliminary qualitative phytochemical screening of the leaves of $C$. esculenta. The preliminary qualitative phytochemical screening of the leaves proved it to contain an array of several important phytochemicals of medicinal importance, including flavonoids, saponins, tannins and glycosides. Some of these phytochemicals have been reported to have several medicinal effects, including antioxidant, hepatoprotective, antiproliferative and antimicrobial properties.

Table 1. Mean values of lipids in rats fed experimental feed containing extract of C. esculenta versus baseline and control.

\begin{tabular}{|c|c|c|c|c|c|}
\hline \multirow{2}{*}{ Group } & \multicolumn{5}{|c|}{ Mean Concentration of Serum Lipid \pm SD $(\mathrm{mmol} / \mathrm{L})$} \\
\hline & Total Cholesterol & $T A G$ & $L D L$ & $H D L$ & $V L D L$ \\
\hline Baseline & $3.39 \pm 0.97$ & $3.85 \pm 0.83$ & $1.79 \pm 0.92$ & $0.83 \pm 0.26$ & $0.77 \pm 0.29$ \\
\hline Control & $4.01 \pm 2.28$ & $0.22 \pm 0.18$ & $1.53 \pm 1.19$ & $3.00 \pm 1.64$ & $0.04 \pm 0.03^{\mathrm{a}^{\mathrm{T}}}$ \\
\hline Test & $0.31 \pm 0.25^{\mathrm{a}^{*}}$ & $2.39 \pm 0.84$ & $0.07 \pm 0.04^{\mathrm{a}^{*}}$ & $0.22 \pm 0.12$ & $0.46 \pm 0.16$ \\
\hline
\end{tabular}

Table 1 above showing the mean values in $\mathrm{mmol} / \mathrm{L}$ for Total Cholesterol, Triacylglycerols (TAG), Low density Lipoprotein (LDL), High Density Lipoprotein (HDL), and Very Low Density Lipoproteins (VLDL) in the three groups of rats. Values with same superscript $\left(^{a^{*}}\right)$ are significantly different $(p<0.05)$.

Table 2. Qualitative phytochemical screening of leaves of C. esculenta.

\begin{tabular}{ll}
\hline Phytochemical & Inference \\
\hline Alkaloids & + \\
Flavonoids & + \\
Saponins & + \\
Cardiac glycosides & + \\
Tannins & + \\
Sterols & + \\
Reducing sugars & - \\
Terpenoids & + \\
Phenols & + \\
\hline
\end{tabular}

Key: - = Not detected $+=$ sparingly present $;++=$ moderately present $;+++$ $=$ highly present.

\section{Discussion}

Lipids are naturally occurring substances required in our diets to supply the body with molecules involved in many cellular functions including serving as components in cell membranes, maintaining membrane fluidity, production of hormones as well as energy reserves [20]. It is important, then that the body's supply of lipids be adequate and regulated. However, when blood lipids rise above the normal threshold, they tend to have deleterious effects especially as it is implicated in the onset of atherosclerosis, which is the leading cause of death attributed to CVDs in developed countries [1] and more recently, in developing nations. Current management remedies of lowering high blood lipid levels include changes in lifestyle trends, exercise and dietary measures. In the light of emerging efforts aimed at reducing risk of CVDs through the regulation of blood lipid levels, therapeutic benefits of plant foods have received much attention as the focus of many dietary studies [21]. Traditional plants have remained alternative sources in treatment of diseases in ethnomedicine but few have been substantiated scientifically, thus the need to study the effects of supplementing $C$. esculenta leaf extracts in diets of male Albino rats on their serum lipid profile. The increase in the triacylglycerol and VLDL levels of test group may indicate a hypertriacylglycerolaemic effect of $C$. esculenta leaves. This effect may be due to the activation and enhancement of fatty acid and TAG synthesis. It is noteworthy to explain that the increases in TAG and VLDL levels in the test did not rise up to the levels obtained for the baseline which indicates the normal or steady state status. It could therefore be reported that the extracts of $C$. esculenta leaves raised TAG and VLDL to a safe or steady state level.

A decrease in the level of LDL-cholesterol was also observed in the test and control of $0.072 \mathrm{mmol} / \mathrm{L}$ and $1.593 \mathrm{mmol} / \mathrm{L}$ respectively as compared to the baseline value of $1.790 \mathrm{mmol} / \mathrm{L}$ which was significantly different $(p<0.05)$. Coronary risk is well established by the elevated levels of total cholesterol especially LDL-cholesterol [22] thus the need to evaluate $C$. esculenta leaves commonly consumed in sauces for coronary risk lowering effects.

The LDL value obtained for the test group was significantly lower $(p<0.05)$ that the control implying that $C$. 
esculenta leaf extract has a tendency to suppress the development of CVDs by lowering serum cholesterol level. A similar trend was obtained for $\mathrm{TC}$ as the leaves of $C$. esculenta reduced was obtained for TC as the leaves of $C$. esculenta reduced the TC in the test significantly $(p<0.05)$ over the control and baseline accentuating its cardioprotective effect.

In this study, the significant decrease in serum lipid profile levels observed with $C$. esculenta may presumably be mediated through a control of lipid metabolism by some of the reported phytochemicals present in the plant.

Flavonoids prevent the oxidation of low-density lipoprotein, lowers the blood levels of cholesterol and triglycerides thereby reducing the risk for the risk for the development of atherosclerosis [23]. As reported by this study, phytochemical screening showed that the leaves of $C$. esculenta had moderate presence of flavonoids. They have also been reported to have vaso-dilatory and inhibitory effects on platelet aggregation thereby preventing coronary heart [24]. The lowering effects of the ethanol extracts of the leaves of $C$. esculenta on total cholesterol may be attributed to its flavonoid content.

This study investigated for the presence of saponins and inferred that it was present. Saponins have been reported to have beneficial effects on blood cholesterol levels. They bind to bile salts and cholesterol in the intestinal tract and cause a reduction of blood cholesterol by preventing its reabsorption. The non-sugar part of saponins also has antioxidant activity which may help to reduce risk of heart diseases [25]. The findings of this work corroborates the findings of other studies which evaluated the effects of some medicinal plants used in traditional settings in which a reduction in lipid profile of experimental animals were drastically reduced.

Cardiac glycosides have been variously used from time immemorial as diuretics and heart tonics due to their beneficial effect on the heart. Cardiac sterols are important components of modern pharmaceuticals employed in the treatment of congestive heart failure and atrial fibrillation. They increase the force of contraction of the heart and are very useful for heart failure patients. They act by affecting the availability of intracellular $\mathrm{Ca}^{2+}$ for myocardial contraction or increasing the sensitivity of myocardial contractile proteins [26].

This study also demonstrated the presence of terpenoids. Terpenoids occur in nearly every natural food and have been associated with protection from and treatment of heart disease due to their antioxidant properties [27]. The findings of its presence in the leaf extract may also be linked to the overall effect of the plant in lowering blood lipids levels in the animals used for the study.

The observed significant reduction in serum total lipids, total cholesterol and LDL cholesterol by the extract can be attributed to the presence of secondary metabolites in the plant which suggests its use in the management of cardiovascular ailments due to hyperlipidemia [28].

\section{Conclusion}

In conclusion, this work demonstrates that the leaves of $C$. esculenta may play an important role in lowering serum levels of total cholesterol and LDL implicated in promoting atherosclerotic syndrome and certain disease processes. It may also be important in maintaining lipid balance between the different lipid fractions in the serum. Significantly, this study discovered that $C$. esculenta can be beneficial for the control and management of cardiac complications arising from hyperlipidemia with concomitant identification of phytochemicals that may be responsible for its action. Since the plant (especially the corm) is edible in this part of South Eastern Nigeria, this is a step in the right direction as incident cases of CVDs linked to high levels of serum lipids may be mitigated by encouraging its mere consumption and further inclusion in the fare of the general populace (this may aid food security on the long run). Grey areas that have remained a mystery in the onset of blood-linked cardiovascular diseases may be explained with further work using similar or related plants. This investigation therefore represents a novel effort for the development of nutraceuticals or new pharmaceuticals for the treatment or management of CVDs. Further studies on the plant leaves may be to isolate and characterize the bioactive phytochemicals responsible for the observed effects of the plant.

\section{Ethical Approval}

The animals were handled following standard ethical guidelines on animal handling and research.

\section{Competing Interests}

Authors have declared that no competing interests exist.

\section{Authors' Contributions}

This work was carried out in collaboration between all authors. Author Frank Uchenna Eneh designed and supervised the study. Author Chibuike Johnson Ogbu performed the experimental analyses. Authors Frank Uchenna Eneh and Chibuike Johnson Ogbu managed the analyses of the study and the literature searches. Author Uchechukwu Chibuzo Ogbodo wrote the protocol and the first draft of the manuscript. Authors Frank Uchenna Eneh and Uchechukwu Chibuzo Ogbodo read and approved the final manuscript.

\section{Acknowledgements}

The authors would like to thank the editors for submitting their insightful research for publication and the reviewers for their important input and critiques to improve the manuscripts. 


\section{References}

[1] WHO (2017). Fact sheet on Cardiovascular disease.

[2] Xenouis, P. G. and Steiner, J. M. (2010). Lipid metabolism and hyperlipidemia in dogs. Vet. J. 183: 12-21.

[3] Adams, K. F., Schatzkin, A., Harris, T. B., Kipnis, V., Mouw, T., Ballard-Barbash, R. et al. (2006). Overweight, obesity, and mortality in a large prospective cohort of persons 50 to 71 years old. N. Engl. J. Med. 355 (8): 763-78.

[4] Pischon, T., Nöthlings, U. and Boeing, H. Obesity and cancer (2008). Proc. Nutr. Soc. 67 (2): 128-45.

[5] Eneh, F. U., Ugochukwu, C. G. and Okoye, C. M. (2018). Effect of Ethanol extract of Curcubita pepo leaves on the Lipid Profile of Wistar Albino Rats. Asian J. Res. Biochem. 2 (4): 1-7.

[6] Abuajah, C. I., Ogbonna, A. C. and Osuji, C. M. (2015). Functional components and medicinal properties of food: A review. J. Food Sci. Tech. 52: 2522-2529.

[7] Krishnapriya, T. V. and Suganthi, A. (2017). Biochemical and phytochemical analysis of Colocasia esculenta (L.) Schott tubers. Int. J. Res. Pharma. \& Pharmaceu. Sci. 2 (3): 21-25.

[8] Yadav, M. Kushawaha, D. K. Chatterji, S. and Watal, G. (2017). Assessment of Antioxidant Activity and Phytochemical Screening of Colocasia esculenta Corm. Int. J. Pharma Sci. Res. 32: 1758-64.

[9] Grindley, B. A., Omoruyi, F., Asemota, H. N. and Morrisona, A. (2002). Carbohydrate digestion and intestinal ATPases in streptozotocin-induced diabetic rats fed extract of yam (Dioscorea cayenesis) or dasheen (Colocasia esculenta). Nutr. Res. 22: 333-341.

[10] Brown, A. C., Reitzenstein, J. E., Liu, J. and Jadus, M. R. (2005). The anti-Cancer effects of poi (Colocasia esculenta) on colonic adenocarcinoma cells in vitro. Physio. Res. 19 (9): 767-71.

[11] Sakano, Y., Mutsuga, M., Tanaka, R., Suganuma, H., Inakuma, T., Tovoda, M. et al. (2005). Inhibition of Human Lanosterol synthase by the constituents of Colocasia esculenta (Taro). Biol. \& Pharma. Bull. 28: 299-304.

[12] Boban, P. T., Nambisan, B. and Sudhakaran, P. R. (2006). Hypolipidaemic effect of chemically different mucilages in rats: A comparative study. Brit. J. Nutr. 96: 1021-1029.

[13] Shah, B. N., Nayak, B. S., Bhatt, S. P., Jalalpure, S. S. and Sheth, A. K. (2007). The anti-inflammatory activity of the leaves of Colocasia esculenta. Saudi Pharma. J. 15: 3-4.

[14] Ufelle, S. A., Onyekwelu, K. C., Ghasi, S., Ezeh, C. O., Ezeh, R. C. and Esom, E. A. (2018). Effects of Colocasia esculenta leaf extract in anemic and normal Wistar rats. J. Med. Sci. 38: 102-106.
[15] Azubuike, N. C., Okwuosa, C. N., Nwachukwu, D. C., Onyemelukwe, A. O., Onukwe, O. S., Chukwu, I. J. P., Orji, O., Orjiakor, N. P. and Achukwu, P. U. (2017). In vivo hepatoprotective studies on saponin and alkaloid rich fractions isolated from Colocasia esculenta (L. schott) leaves Pharmacol. Online 2: 66-74.

[16] Keshav, A., Sharma, A. and Mazumdar, B. (2019). Phytochemical Analysis and Antioxidant Activity of Colocasia esculenta (L.) leaves. Int. J. Chem. \& Mol. Eng. 13 (1): 20-23.

[17] Kalariya, M., Parmar, S. and Sheth, N. (2010). Neuropharmacological activity of hydroalcoholic extract of leaves of Colocasia esculenta. Pharma. Biol. 201: 48: 12071212 .

[18] Sidhu, D. and Naugler, C. (2012). Fasting time and lipid levels in a community-based population: a cross-sectional study. Arch. Intern. Med. 172: 1707-1710.

[19] Harborne, J. P. (1973). Phytochemical methods. London. Chapman and Hall Limited, pp. 49-88.

[20] Das, U. N. (2006). Essential fatty acids: biochemistry, physiology and pathology. Biotech. J. 1 (4): 420-439.

[21] Enkhmaa, B., Surampudi, P., Anuurad, E. and Berglund, L. (2000). Lifestyle Changes: Effect of Diet, Exercise, Functional Food, and Obesity Treatment on Lipids and Lipoproteins. [Updated 2018 Sep 11]. In: Feingold KR, Anawalt B, Boyce A, et al., editors. Endotext [Internet]. South Dartmouth (MA): MDText.com, Inc.

[22] Bagri, P., Ali, M., Aeri, V., Bhowmik, M. and Sultana, S. (2009). Antidiabetic effect of Punica granatum flowers: effect on hyperlipidemia, pancreatic cells lipid peroxidation and antioxidant enzymes in experimental diabetes. Food \& Chem. Toxic. 47: 50-54.

[23] Subramani, S. and Casmir, C. A. (2002). Flavonoids and antioxidant activity of Georgia grown Vidalia onions. J. Agric. Food Chem. 50 (19): 5338-5342.

[24] Okwu, D. E. (2001). Evaluation of the chemical composition of indigenous spices and flavoring agents. Glob. J. Pure Appl. Sci. 7 (3): 455-459.

[25] Oakenfull, D. and Sidhu, G. (1990). Could saponins be a useful treatment for hypercholesterolemia. Euro. J. Clin. Nutr. 44: 79-88.

[26] Walker, A. F., Marakis, G., Morris, A. P. and Robinson, P. A. (2002). Promising hypotensive effect of hawthorn extract: a randomized double-blind pilot study of mid, essential hypertension. Phytoth. Res. 16 (1): 48-54.

[27] Wagner, K. H. and Elmadfa, I. (2003). Biological relevance of terpenoids. Overview focusing on mono-, di- and tertraterpenes. Anna. Nutr. Met. 47 (3): 95-106.

[28] Kwiterovich, P. O. (2000). The metabolic pathways of highdensity lipoprotein, low-density lipoprotein and triglycerides: a current review. Am. J. Cardiol. 12: 510. 